This information is current as of April 26, 2023.

\title{
Diagnostic Accuracy of CT Angiography and CT Perfusion for Cerebral Vasospasm: A Meta-Analysis
}

E.D. Greenberg, R. Gold, M. Reichman, M. John, J. Ivanidze, A.M. Edwards, C.E. Johnson, J.P. Comunale and P. Sanelli

AJNR Am J Neuroradiol 2010, 31 (10) 1853-1860

doi: https://doi.org/10.3174/ajnr.A2246

http://www.ajnr.org/content/31/10/1853 


\section{ORIGINAL RESEARCH}

E.D. Greenberg

R. Gold

M. Reichman

M. John

J. Ivanidze

A.M. Edwards

C.E. Johnson

J.P. Comunale

P. Sanelli

\section{Diagnostic Accuracy of CT Angiography and CT Perfusion for Cerebral Vasospasm: A Meta-Analysis}

BACKGROUND AND PURPOSE: In recent years, the role of CTA and CTP for vasospasm diagnosis in the setting of ASAH has been the subject of many research studies. The purpose of this study was to perform a meta-analysis of the diagnostic performance of CTA and CTP for vasospasm in patients with ASAH by using DSA as the criterion standard.

MATERIALS AND METHODS: The search strategy for research studies was based on the Cochrane Handbook for Systematic Reviews, including literature data bases (PubMed, Embase, Cochrane Database of Systematic Reviews, and the Web of Science) and reference lists of manuscripts published from January 1996 to February 2009. The inclusion criteria were the following: 1) published manuscripts, 2) original research studies with prospective or retrospective data, 3) patients with ASAH, 4) CTA or CTP as the index test, and 5) DSA as the reference standard. Three reviewers independently assessed the quality of these research studies by using the QUADAS tool. Pooled estimates of sensitivity, specificity, LR+, LR-, DOR, and the SROC curve were determined.

RESULTS: CTA and CTP searches yielded 505 and 214 manuscripts, respectively. Ten research studies met inclusion criteria for each CTA and CTP search. Six CTA and 3 CTP studies had sufficient data for statistical analysis. CTA pooled estimates had $79.6 \%$ sensitivity $(95 \% \mathrm{Cl}, 74.9 \%-83.8 \%), 93.1 \%$ specificity $(95 \% \mathrm{Cl}, 91.7 \%-94.3 \%), 18.1 \mathrm{LR}+(95 \% \mathrm{Cl}, 7.3-45.0)$, and $0.2 \mathrm{LR}-(95 \% \mathrm{Cl}, 0.1-0.4)$; and CTP pooled estimates had $74.1 \%$ sensitivity $(95 \% \mathrm{Cl}, 58.7 \%-86.2 \%), 93.0 \%$ specificity $(95 \% \mathrm{Cl}$, 79.6\%-98.7\%), 9.3 LR+ (95\% Cl, 3.4-25.9), and 0.2 LR- (95\% Cl, 0.04-1.2). Overall DORs were $124.5(95 \% \mathrm{Cl}, 28.4-546.4)$ for CTA and 43.0 (95\% Cl, 6.5-287.1) for CTP. Area under the SROC curve was $98 \pm 2.0 \%$ for CTA and $97 \pm 3.0 \%$ for CTP

concLusions: The high diagnostic accuracy determined for both CTA and CTP in this meta-analysis suggests that they are potentially valuable techniques for vasospasm diagnosis in ASAH. Awareness of these results may impact patient care by providing supportive evidence for more effective use of CTA and CTP imaging in ASAH.

ABBREVIATIONS: $\mathrm{ASAH}=$ aneurysmal subarachnoid hemorrhage; $\mathrm{AUC}=$ area under the curve;
$\mathrm{CBF}=$ cerebral blood flow; $\mathrm{CI}=$ confidence interval; $\mathrm{CTA}=\mathrm{CT}$ angiography; $\mathrm{CTP}=\mathrm{CT}$ perfusion;
$\mathrm{DCI}=$ delayed cerebral ischemia; $\mathrm{DOR}=$ diagnostic OR; $\mathrm{DSA}=$ digital subtraction angiography; ICA =
internal carotid artery; $\mathrm{LR}=$ likelihood ratio; $\mathrm{LR}-=$ negative likelihood ratio; $\mathrm{LR}+=$ positive likelihood
ratio; MeSH = Medical Subject Headings; $\mathrm{MTT}=$ mean transit time; NA = not applicable; NIH =
National Institutes of Health; NINDS = National Institute of Neurological Disorders and Stroke; NPV =
negative predictive value; OR = odds ratio; PPV = positive predictive value; QUADAS = quality
assessment of diagnostic accuracy studies; $\mathrm{ROI}=$ region of interest; SROC = summary receiver
operating characteristic analysis; STARD = standards for reporting of diagnostic accuracy

A neurysmal subarachnoid hemorrhage is a devastating condition affecting as many as 30,000 Americans each year. ${ }^{1}$ Despite many advances in the diagnosis and treatment

Received March 10, 2010; accepted after revision May 30.

From the Department of Radiology (E.D.G., M.R., M.J., C.E.J., J.P.C., P.S.), Weill Cornell Medical College, New York-Presbyterian Hospital, New York, New York; New York College of Osteopathic Medicine (R.G.), Old Westbury, New York; Department of Public Health (M.J., A.M.E., P.S.), Weill Cornell Medical College, New York, New York; and Ludwig Maximilians University of Munich (J.I.), Munich, Germany.

This work was supported by grant 5K23NS058387-02 from the NINDS, a component of the $\mathrm{NIH}$. The contents of this work are solely the responsibility of the authors and do not necessarily represent the official view of NINDS or NIH.

Paper previously presented in part at: Annual Meeting of the Eastern Neuroradiological Society, August 12-14, 2010, Washington, DC; and the Annual Meeting of the American Society of Neuroradiology, May 15-20, 2010, Boston, Massachusetts.

Please address correspondence to Edward D. Greenberg, MD, Department of Radiology, Weill Cornell Medical College, New York-Presbyterian Hospital, 1320 York Ave, 32E, New York, NY, 10021; e-mail: edg2008@nyp.org

Indicates open access to non-subscribers at www.ajnr.org

DOI 10.3174/ajnr.A2246 of ASAH, outcomes for patients remain poor, with mortality rates as high as $45 \%-50 \%$ and significant morbidity among many of the survivors. ${ }^{1,2}$ One of the major causes of morbidity and mortality in the setting of ASAH is cerebral vasospasm, with the sequelae of permanent neurologic deficit, infarction, and death. ${ }^{3}$

Cerebral vasospasm following ASAH is a complex entity that can be seen in $\leq 70 \%$ of patients with ASAH, with typical onset at 3-5 days after hemorrhage and maximal narrowing occurring at 5-14 days on DSA. ${ }^{1}$ However, not all patients with angiographic vasospasm manifest clinical symptoms. DCI or symptomatic vasospasm affects $20 \%-30 \%$ of patients with ASAH. The caveat is that patients do not necessarily have both clinical and imaging findings of vasospasm and symptoms can be nonspecific. ${ }^{4}$ Therefore, more accurate and reliable methods are being investigated for detecting vasospasm. In the past few years, emerging technology such as CTA and CTP has been the focus of many research studies published in the literature. However, it is challenging to draw clear and 
confident conclusions from these research studies, given the variability in their methodologic quality and results. Most important, this variability may impact the management of patients with ASAH, resulting in overuse of CTA and CTP imaging at institutions that consider these modalities as having high diagnostic accuracy compared with other institutions that may underuse CTA and CTP on the basis of studies reporting lower accuracy.

In the practice of evidence-based medicine, searching the literature for information on new techniques in patients with ASAH is an essential step. Given the radiation exposure risks and the increased costs associated with CTA and CTP imaging, establishing its value has become increasingly important for determining appropriate use guidelines in specific patient populations. The purpose of this meta-analysis is to evaluate the sensitivity and specificity of CTA and CTP for vasospasm diagnosis in patients with ASAH, by using DSA as the criterion standard.

\section{Materials and Methods}

Two comprehensive literature searches were performed by using both electronic and manual methods by an author (E.D.G.) along with 2 experienced medical librarians, both of whom had masters in library and information science degrees with 25 years' and 15 years' experience. In the first search, all studies that evaluated the diagnostic performance of CTA for cerebral vasospasm in patients with ASAH by using DSA as the reference standard were identified. The second search focused on CTP compared with DSA for vasospasm diagnosis. The search strategy was performed according to the Cochrane Handbook for Systematic Reviews. ${ }^{5}$ The electronic literature search included publications printed in the English language from January 1996 through February 2009. The search was performed in PubMed, Embase, Cochrane, and the Web of Science data bases. In designing the electronic search, the terminology used was based on the standardized National Library of Medicine MeSH as well as free text for all components of our search, including CT angiography, CT perfusion, subarachnoid hemorrhage, and vasospasm. Tables 1 and 2 show the search terminology used.

An author (E.D.G.) closely reviewed the search results with the medical librarians and selected relevant manuscripts for this metaanalysis on the basis of the titles and abstracts. The "Related Articles" feature in PubMed was also used for all the selected research studies to maximize the probability of finding additional relevant studies. The full text of the selected manuscripts was reviewed by 3 authors (E.D.G., M.R., R.G.) to determine inclusion in this meta-analysis. Disagreements were resolved by consensus. After the relevant research studies were identified, the reference list of each manuscript was manually searched for additional relevant studies. Relevant research studies included in this meta-analysis were those that assessed the diagnostic accuracy of CTA or CTP for cerebral vasospasm compared with DSA in patients with ASAH. The inclusion criteria for relevant research studies were the following: 1) published manuscripts, 2) original research studies with prospective or retrospective data, 3) patients with ASAH, 4) CTA or CTP as the index test, and 5) DSA as the criterion standard. The exclusion criteria were the following: 1) abstracts only, 2) review articles, 3) nonhuman models (animal or phantom), and 4) non-English publications.
Table 1: Search methods for identification of CTA research studies

\section{Electronic Searches}

1) MEDLINE (through PubMed)

"Tomography, x-ray computed" (MeSH Terms) OR "tomography" (All Fields) AND "x-ray" (All Fields) AND "computed" (All Fields) OR "x-ray computed tomography" (All Fields) OR "tomography, x-ray computed" (All Fields) OR "CT" (All Fields) AND "subarachnoid haemorrhage" (All Fields) OR "subarachnoid hemorrhage" (MeSH Terms) OR "subarachnoid" (All Fields) AND "hemorrhage" (All Fields) OR "subarachnoid hemorrhage" (All Fields) AND "angiography" (MeSH Terms) OR "angiography" (All Fields) AND "Vasoconstriction" (Mesh) OR "Vasospasm, Intracranial" (Mesh) OR "vasoconstriction" (MeSH Terms) OR "vasoconstriction" (All Fields) OR "vasospasm" (All Fields) AND "humans" (MeSH Terms) AND English (lang) AND "aneurysm" (MeSH Terms) OR "aneurysm" (All Fields) AND "humans" (MeSH Terms) AND English (lang)

2) Embase (via OVID)

ct.mp. or exp Computer-Assisted Tomography/AND angiography.mp. AND subarachnoid hemorrhage.mp. or exp Subarachnoid Hemorrhage/AND exp Vasospasm/or exp Brain Vasospasm/or vasospasm.mp. AND exp Intracranial Aneurysm/or ANEURYSM.mp. or exp Aneurysm

$\mathrm{mp} .=$ title, abstract, subject headings, heading word, drug trade name, original title, device manufacturer, drug manufacturer's name

Search terms: aneurysm, angiography, assisted, brain, computer, hemorrhage, intracranial, subarachnoid, tomography, vasospasm

3) Cochrane

Subarachnoid Hemorrhage AND Angiography AND Vasospasm AND Aneurysm AND CT in "Title, Abstract, or Keywords"

4) Web of Science:

Topic $=($ CT) AND Topic $=($ Subarachnoid Hemorrhage $)$ AND Topic $=$ $($ Aneurysm) AND Topic $=($ vasospasm) AND Topic $=($ angiography)

Table 2: Search methods for identification of CTP research studies

\section{Electronic Searches}

1) MEDLINE (through PubMed)

"Contraindications" (Subheading) OR "contraindications" (All Fields) OR "CT" (All Fields) OR CTP (All Fields) OR "tomography, x-ray computed" (MeSH Terms) OR "tomography" (All Fields) AND "x-ray" (All Fields) AND "computed" (All Fields) OR "x-ray computed tomography" (All Fields) OR "tomography, x-ray computed" (All Fields) AND "vasospasm, intracranial" (MeSH Terms) OR "vasospasm" (All Fields) AND

"intracranial" (All Fields) OR "intracranial vasospasm" (All Fields) OR "vasospasm" (All Fields) AND "intracranial" (All Fields) OR "vasospasm, intracranial" (All Fields) AND "perfusion" (MeSH Terms) OR "perfusion" (All Fields)

2) Embase (via OVID)

CT.mp. or Computer Assisted Tomography AND Vasospasm/or Brain Vasospasm/or vasospasm.mp. AND Brain Perfusion/or perfusion.mp. or Perfusion. LIMIT TO ENGLISH AND HUMANS ( $\mathrm{mp}=$ title, abstract, subject headings, heading word, drug trade name, original title, device manufacturer, drug manufacturer's name)

3) Cochrane: CT OR Computed Tomography AND Perfusion AND Vasospasm

4) Web of Knowledge: Topic $=$ (computed tomography) AND Topic $=$ (perfusion) AND Topic $=($ vasospasm)

\section{Data Extraction}

In general, "vasospasm" was defined on CTP as a perfusion deficit demonstrating prolonged MTT and decreased CBF. There were no standardized thresholds for CBF and MTT to determine vasospasm, thus contributing to the heterogeneity among these studies. "Angiographic vasospasm" was defined as evidence of arterial narrowing compared with the parent vessel or with a baseline examination, with both symptomatic and asymptomatic patients included.

The QUADAS tool was used to assess the methodologic quality of each study included in the meta-analysis to identify study bias and 


\begin{tabular}{|c|c|c|c|c|}
\hline \multicolumn{5}{|c|}{ Table 3: QUADAS tool } \\
\hline Item & & Yes & No & Unclear \\
\hline 1) & Was the spectrum of patients representative of the patients who will receive the test in practice? & (1) & () & (1) \\
\hline 2) & Were selection criteria clearly described? & () & () & () \\
\hline 3) & Is the reference standard likely to correctly classify the target condition? & (1) & () & () \\
\hline 4) & $\begin{array}{l}\text { Is the time period between reference standard and index test short enough to be reasonably sure } \\
\text { that the target condition did not change between the } 2 \text { tests? }\end{array}$ & (1) & () & () \\
\hline 5) & $\begin{array}{l}\text { Did the whole sample or a random selection of the sample receive verification using a reference } \\
\text { standard of diagnosis? }\end{array}$ & () & () & () \\
\hline 6) & Did patients receive the same reference standard regardless of the index test result? & () & () & () \\
\hline 7) & $\begin{array}{l}\text { Was the reference standard independent of the index test (ie, the index test did not form part of } \\
\text { the reference standard)? }\end{array}$ & () & (1) & () \\
\hline 8) & $\begin{array}{l}\text { Was the execution of the index test described in sufficient detail to permit replication of the } \\
\text { test? }\end{array}$ & () & () & () \\
\hline 9) & $\begin{array}{l}\text { Was the execution of the reference standard described in sufficient detail to permit its } \\
\text { replication? }\end{array}$ & () & () & () \\
\hline 10) & $\begin{array}{l}\text { Were the index test results interpreted without knowledge of the results of the reference } \\
\text { standard? }\end{array}$ & () & () & (1) \\
\hline 11) & $\begin{array}{l}\text { Were the reference standard results interpreted without knowledge of the results of the index } \\
\text { test? }\end{array}$ & () & () & () \\
\hline 12) & $\begin{array}{l}\text { Were the same clinical data available when test results were interpreted as would be available } \\
\text { when the test is used in practice? }\end{array}$ & () & () & () \\
\hline 13) & Were uninterpretable/intermediate test results reported? & () & () & () \\
\hline 14) & Were withdrawals from the study explained? & () & () & () \\
\hline
\end{tabular}

limitations. ${ }^{6}$ The QUADAS tool is a structured list of 14 questions (Table 3). These questions evaluate the study design, sample size, and bias and limitations in the research studies. For example, QUADAS item 4 asks, "Is the time period between reference standard and index test short enough to be reasonably sure that the target condition did not change between the 2 tests?" A period of 12 hours was defined on the basis of several studies in the literature that have demonstrated vasospasm as a dynamic process that varies in disease severity during a time interval of 48 or 72 hours. ${ }^{7,8}$ Additionally, the clinical manifestations of delayed cerebral ischemia evolve gradually, during several hours. $^{2}$ An ideal comparison of CTA/CTP with DSA for vasospasm diagnosis would involve performing these examinations serially and as close together in time as possible. Given the practical aspects of performing and interpreting these examinations in patients in the neuro-intensive care unit, a 12 -hour period was considered as a short enough time interval between the index test (CTA or CTP) and the criterion standard (DSA) to be reasonably sure that the target condition (vasospasm) did not change between the 2 tests. Two blinded reviewers (M.R. and R.G.) performed the data extraction and QUADAS scoring for each research study. A third reviewer (P.S.) served as a blinded expert in cases of disagreement.

The statistical data extraction was performed by 1 reviewer (J.I.) for each research study. The data were recorded in the form of a $2 \times 2$ table if the actual data (true-positives, true-negatives, false-positives, and false-negatives) were reported. However, if these data were not mentioned, then statistical data in the form of sensitivity, specificity, PPV, and NPV were recorded. Additional data were recorded from research studies that described the statistical analysis according to arterial segments, either distal or proximal. In our analysis, the proximal vessels were defined as the first-order arterial segments, including the ICA, the A1 segment of the anterior cerebral artery, the M1 segment of the middle cerebral artery, the anterior communicating artery, the vertebral artery, the basilar artery, the P1 segment of the posterior cerebral artery, and the posterior communicating artery. The distal vessels were defined as the second-order arterial segments, such as the A2, M2, and P2 segments.

\section{Statistical Analysis}

The pooled sensitivity, specificity, LR,$+ \mathrm{LR}-$, and DOR with the corresponding 95\% CIs were calculated. ${ }^{9,10}$ The pooled indices of sensitivity and specificity were calculated by using weighted averages according to the sample size of each study. The pooled estimates of LRs and DORs were computed by the DerSimonian and Laird method based on a random-effects model. ${ }^{11}$ Forest plots and statistical analysis by using the LR test, the Cochran $\mathrm{Q}$, and the inconsistency index were performed to evaluate the threshold effect as an important component of the source of variation of the diagnostic studies used in the different research studies. The inconsistency index describes the percentage of total variance due to heterogeneity rather than chance across these different studies. A zero percentage index indicates that there is no heterogeneity, whereas $25 \%, 50 \%$, and $75 \%$ indices correspond to low, moderate, and high heterogeneity, respectively. ${ }^{12}$ Given the presence of diagnostic threshold variation in these research studies, the SROC curve is an appropriate summary statistic to assess the overall diagnostic accuracy of CTA or CTP across the different threshold definitions. Therefore, the results are described as the area under the SROC curve with its $Q^{*}$-point representing the maximal joint sensitivity and specificity. ${ }^{13,14}$ Furthermore, the shape of the SROC curve (symmetric versus asymmetric) was determined by assessing the changes in DOR according to diagnostic thresholds by using the Moses-Shapiro-Littenberg method. ${ }^{15}$ Statistical analyses were performed by 2 biostatisticians (M.J., A.M.E.) by using Meta-DiSc software, Version 1.4 (Clinical Biostatistics Unit, Hospital Ramon y Cajal, Madrid, Spain). ${ }^{16}$

\section{Results}

The first search that evaluated the diagnostic accuracy of CTA for vasospasm yielded a total of 505 manuscripts. Most of these manuscripts were not considered relevant for this metaanalysis due to the broad search strategy capturing all research studies with the terms "vasospasm" and "subarachnoid hemorrhage." Manual searching of the reference lists of the relevant research studies did not yield any additional relevant 


\begin{tabular}{|c|c|c|c|c|c|c|c|}
\hline Authors & Patients & Segments & Sensitivity & Specificity & PPV & NPV & Accuracy \\
\hline Anderson et $\mathrm{al}^{17} 2000^{\mathrm{a}, \mathrm{b}}$ & 17 & 172 & $57,64,100$ & $93,97,100$ & $54,58,100$ & $93,98,100$ & $88,95,100$ \\
\hline Binaghi et $\mathrm{al}^{18} 2007^{\mathrm{b}}$ & 27 & 286 & 87.7 & 99.2 & 98.3 & 94.1 & 95.4 \\
\hline Chaudhary et $\mathrm{al}^{19} 2008^{\mathrm{b}}$ & 33 & 886 & 63 & 90 & 43 & 95 & 87 \\
\hline Joo et $\mathrm{al}^{20} 2006$ & 8 & 24 & NA & NA & NA & NA & NA \\
\hline Ko et $\mathrm{al}^{21} 2003$ & 6 & 30 & 60 & 100 & NA & 37 & NA \\
\hline Ochi et al ${ }^{22} 1997$ & 2 & 10 & NA & NA & NA & NA & NA \\
\hline Otawara et $a^{23} 2002^{b}$ & 20 & 154 & NA & NA & NA & NA & NA \\
\hline Takagi et $\mathrm{al}^{24} 1998$ & 13 & NA & NA & NA & NA & NA & NA \\
\hline Wintermark et $\mathrm{al}^{25} 2006^{\mathrm{b}}$ & 27 & 123 & 75.6 & 95.3 & 79.5 & 94.2 & 91.4 \\
\hline Yoon et $a^{26} 2006^{b}$ & 17 & 251 & 97.5 & 98.1 & 90.7 & 99.5 & 98 \\
\hline
\end{tabular}

a Values given for mild, moderate, and severe vasospasm groups, respectively.

$\mathrm{b}$ The study was included in the statistical calculations of the meta-analysis.

\begin{tabular}{|c|c|c|c|c|c|c|c|c|}
\hline Authors & Patients & $\begin{array}{l}\text { CTP } \\
\text { Scans }\end{array}$ & $\begin{array}{c}\text { Arterial Segments/ } \\
\text { ROls }\end{array}$ & Sensitivity & Specificity & PPV & NPV & Accuracy \\
\hline Binaghi et al ${ }^{18} 2007^{\mathrm{b}, \mathrm{c}}$ & 23 & 23 & NA & 20,90 & 100,100 & 100,100 & $27.3,75$ & 92.3 \\
\hline Harrigan et $a^{27} 2005$ & 10 & 17 & NA & NA & NA & NA & NA & NA \\
\hline Kanazawa el al 202007 & 19 & 26 & 156 & NA & NA & NA & NA & NA \\
\hline Moftakhar et al ${ }^{28} 2006^{\mathrm{C}}$ & 14 & 23 & NA & NA & NA & NA & NA & NA \\
\hline Nabavi et al ${ }^{8} 2001$ & 15 & 59 & 944 ROls & NA & NA & NA & NA & NA \\
\hline Pham et $a^{29} 2006$ & 10 & 36 & NA & NA & NA & NA & NA & NA \\
\hline Sviri et al ${ }^{30} 2006$ & 46 & 63 & NA & 64.5 & 86 & 72.5 & 80.5 & NA \\
\hline Sviri et $\mathrm{a}^{31} 2006$ & 35 & 35 & $420 \mathrm{ROIs}$ & NA & NA & NA & NA & NA \\
\hline Wintermark et al ${ }^{33} 2008$ & 33 & 40 & 200 & 92 & 86 & 79 & 95 & NA \\
\hline Wintermark et al ${ }^{25} 2006^{c}$ & 27 & 35 & 630 & 95.1 & 90.7 & 71.3 & 98.7 & 91.6 \\
\hline
\end{tabular}

a Several studies analyzed the CTP data using arterial segmental anatomy, whereas other studies used ROls.

b Values given for mild-moderate and severe vasospasm groups, respectively.

c The study was included in the statistical calculations of the meta-analysis.

studies. In total, there were 10 research studies included in this meta-analysis (Table 4). ${ }^{17-26}$ However, only 6 of these research studies contained the appropriate data (sample size, sensitivity, specificity, PPV, and NPV) for inclusion in the statistical calculations. ${ }^{17-19,23,25,26}$ The other 4 research studies did not report the actual data from a $2 \times 2$ table or did not report enough data to reconstruct a $2 \times 2$ table. The 6 research studies included in the meta-analysis had a total of 141 patients with 1936 arterial segments analyzed. The mean number of patients in a study was 23.5 (range, 17-33). These research studies were published between 2000 and 2008. The second search that evaluated the diagnostic accuracy of CTP for vasospasm yielded a total of 214 manuscripts. Again, most of these manuscripts were not relevant for this meta-analysis due to our broad search strategy used. Manual searching of the reference lists from these relevant research studies did not yield any additional relevant studies. Only 10 of these research studies were considered relevant for this meta-analysis (Table 5). ${ }^{8,18,25,27-33}$ However, only 3 of these research studies contained the appropriate data for inclusion in the statistical calculations. ${ }^{18,25,28}$ The 3 research studies included in this metaanalysis had a total of 64 patients. The mean number of patients in a study was 21.3 (range, 14-27). The research studies were published between 2006 and 2007.

\section{Quality Assessment}

In the first search that evaluated CTA, the agreement among reviewers by using the QUADAS tool for assessing the methodologic quality of the CTA research studies was $100 \%$ for 10 of the 14 questions, $90 \%$ for questions 8 and 13 , and $80 \%$ for questions 1 and 12. In the second search that evaluated CTP, the agreement among reviewers by using the QUADAS tool to assess the methodologic quality of the CTP research studies was $100 \%$ for 12 of the 14 questions and $90 \%$ for questions 4 and 6. All disagreements were resolved by the third expert reviewer in a blinded fashion. The QUADAS tool revealed several potential sources of bias in the CTA and CTP research studies. Disease-progression bias was possibly present in 6 of the CTA and 6 of the CTP research studies because the time between the criterion standard (DSA) and the index test (CTA) was too long to be reasonably sure that the target condition (vasospasm) did not change between these tests. Review bias may also have been present in 6 of the CTA and 6 of the CTP research studies because the CTA or CTP findings were possibly interpreted with knowledge of the DSA findings. Conversely, 7 of the CTA and 8 of the CTP research studies may have interpreted the DSA with knowledge of the CTA or CTP findings. In addition, 4 of the CTA and 4 of the CTP research studies did not report how uninterpretable/intermediate test results were analyzed, possibly leading to biased assessment of CTA and CTP test characteristics.

\section{Quantitative Analysis}

CTA. The overall range of reported sensitivity and specificity of CTA for detecting vasospasm in patients with ASAH is $63 \%-98 \%$ and $90 \%-98 \%$, respectively. The data from these CTA research studies had high statistical heterogeneity with inconsistency index values of $88.2 \%$ and $87.6 \%$ for sensitivity and specificity (LR test, $P<.001$ ), respectively. Due to this variability in the data, a random-effects SROC model was used by fitting the pooled accuracy data to a single symmetric SROC curve (Fig 1). The area under the SROC curve ( \pm SE) and its 


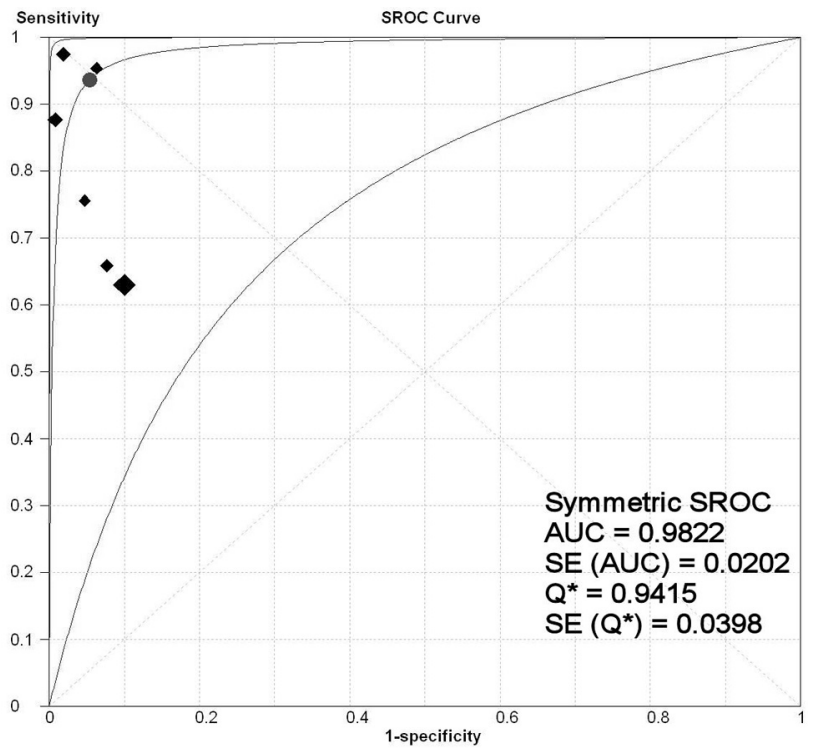

Fig 1. SROC curve for the CTA meta-analysis. Each black diamond represents an individual research study in the CTA meta-analysis, with the size of the diamond directly proportional to the sample size of the study. The best fit curve (middle curve) lies between the other 2 curves, which demarcate its $95 \% \mathrm{Cl}$. The circle denotes the $0^{*}$-point.

$\mathrm{Q}^{\star}$-point $( \pm \mathrm{SE}$ ) were $98 \% \pm 2.0 \%$ and $94 \% \pm 4.0$, respectively. The pooled estimates were $79.6 \%$ sensitivity $(95 \% \mathrm{CI}$, 74.9\%-83.8\%), 93.1\% specificity (95\% CI, 91.7\%-94.3\%), 18.1 LR+ (95\% CI, 7.3-45.0), and 0.2 LR- (95\% CI, 0.10.4). The random-effects model estimated an overall DOR of 124.5 (95\% CI, 28.4-546.4) (Fig 2).

A subanalysis was performed on 5 of the 6 research studies that had sufficient data to evaluate the diagnostic accuracy of CTA for proximal and distal vasospasm. The pooled estimates for proximal vasospasm were $81.7 \%$ sensitivity (95\% CI, 73.2\%$88.4 \%$ ) and $93.7 \%$ specificity (95\% CI, 91.9\%-95.3\%). The area under the SROC curve was $95 \%$. The pooled estimates for distal vasospasm were $85.5 \%$ sensitivity (95\% CI, 77.7\%$91.4 \%$ ) and $92.3 \%$ specificity (95\% CI, 89.4\%-94.6\%). The area under the SROC curve was $99 \%$. Note that the pooled estimates of sensitivity increased for proximal and distal vasospasm compared with the overall sensitivity of $79.6 \%$ due to the omission of 1 research study from the subgroup analysis. All 5 of these research studies included the ICA in their calculations. However, 2 research studies reported separate sensitivity and specificity for the ICA, acknowledging the difficulty in the interpretation of vasospasm due to the surrounding bone. After exclusion of the ICA data from the proximal vasospasm analysis, the pooled sensitivity and specificity were 89.8\% (95\% CI, 82.5-94.8) and 93.3\% (95\% CI, 91.2-95.0), respectively. The area under the SROC was $95 \%$.

CTP. The overall range of reported sensitivity and specificity of CTP for detecting vasospasm in patients with ASAH was $58 \%-95.1 \%$ and $86 \%-100 \%$, respectively. The inconsistency index values for sensitivity and specificity for the CTP research studies were $73.7 \%$ (LR test, $P$ value $=.022$ ) and $0.0 \%$ (LR test, $P$ value $=.469)$, respectively. Note that the $P$ value for the inconsistency index for specificity does not reach statistical significance, indicating that there may be heterogeneity in these data. The area under the SROC curve $( \pm \mathrm{SE})$ and its $\mathrm{Q}^{*}$-point $( \pm \mathrm{SE}$ ) were $97 \% \pm 3.0 \%$ and $91 \% \pm 5.0$. Due to the small sample size in this meta-analysis, the SROC curve is not displayed and it was not possible to calculate the $95 \%$ CI. The pooled estimates for the CTP data were $74.1 \%$ sensitivity (95\% CI, 58.7\%-86.2\%), 93.0\% specificity (95\% CI, 79.6\%98.7\%), 9.3 LR+ (95\% CI, 3.4-25.9), and 0.2 LR - (95\% CI, $0.04-1.2$ ). The overall DOR is 43.0 (95\% CI, 6.5-287.1). Given the small sample size and the heterogeneity in the CTP data, these results are considered initial preliminary findings. A summary of the major quantitative results for CTA and CTP is displayed in Table 6 .

\section{Discussion}

The following are the major points revealed from this metaanalysis: 1) The high diagnostic accuracy determined for both CTA $(98 \pm 2.0 \%)$ and CTP $(97 \pm 3.0 \%)$ are considered initial preliminary findings derived from the current literature, suggesting that CTA and CTP are potentially valuable techniques in patients with ASAH. 2) There are few research studies that met inclusion criteria for this meta-analysis, highlighting the need for future larger prospective studies to be performed. 3) The high variability in methodologic quality and reporting methods demonstrates the importance for future studies to adhere to the STARD criteria to reduce study bias and limitations.

Cerebral vasospasm is the leading cause of morbidity and mortality in patients with ASAH who survive the initial hemorrhage. The term "cerebral vasospasm" is commonly used to refer to both angiographic vasospasm and DCI. "DCI" is defined as either an otherwise unexplained clinical deterioration (ie, a new focal deficit, decrease in level of consciousness, or both) or a new infarct on CT that was not visible on the admission or immediate postoperative scan or both. ${ }^{34}$ "Angiographic vasospasm" is defined as evidence of arterial narrowing compared with the parent vessel or with a baseline examination. Even though DSA is used as the criterion standard in many research studies for evaluating the diagnostic accuracy of CTA and CTP for vasospasm diagnosis, not all patients with angiographic vasospasm manifest clinical symptoms. This difference in vasospasm definitions may impact clinical care. Currently, the diagnosis of vasospasm and management decisions in clinical practice relies on clinical examination, transcranial Doppler sonography, and DSA. Some institutions are also using CTA and CTP to improve their detection of vasospasm. Several research studies in the literature have focused on evaluating CTA and/or CTP for vasospasm diagnosis by comparing with DSA. However, the methodologic quality and results of these studies vary significantly; this variation makes it difficult to compare the quality of studies and accurately summarize the diagnostic performance of CTA and CTP in patients with ASAH. Unfortunately, this difficulty can lead to a lack of consensus in the use of CTA and CTP imaging in clinical practice. Given this variability in the literature, a systematic review and meta-analysis can be helpful in assessing the methodologic quality of these studies and estimating the overall diagnostic accuracy of CTA and CTP compared with DSA.

There are many advantages in performing a meta-analysis, including improved generalizability to the population and the ability to control for study variation. The statistical advantages include greater statistical power to detect an effect and deriva- 


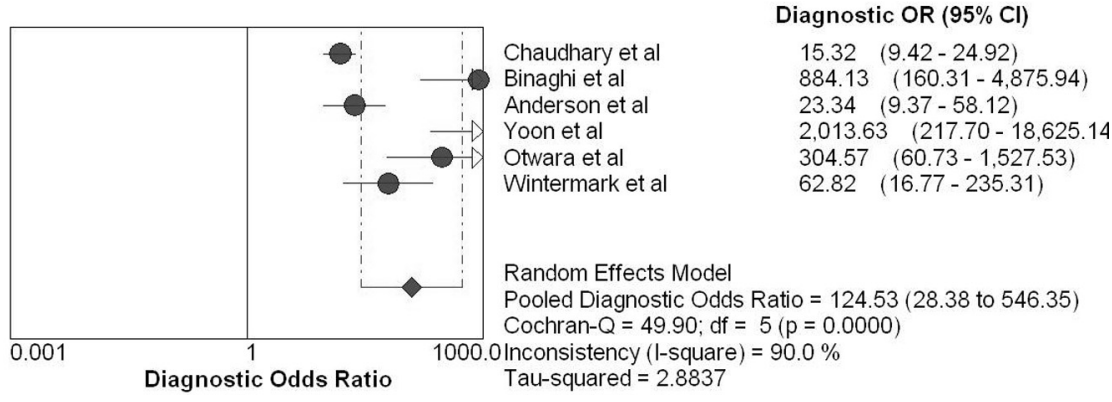

Fig 2. Forest plot of the DOR for the CTA meta-analysis. The DOR for each research study is represented by circles. The 95\% $\mathrm{Cl}$ is represented by the horizontal lines through each circle. The pooled DOR for this meta-analysis is represented by the diamond symbol.

\begin{tabular}{|c|c|c|c|}
\hline & Sensitivity & Specificity & AUC \\
\hline \multicolumn{4}{|l|}{ CTA } \\
\hline All vessels & $79.6 \%$ & $93.1 \%$ & $98.0 \%$ \\
\hline Proximal vessels ${ }^{\mathrm{a}}$ & $89.8 \%$ & $93.3 \%$ & $95.0 \%$ \\
\hline Distal vessels & $85.5 \%$ & $92.3 \%$ & $99.0 \%$ \\
\hline CTP & $74.1 \%$ & $93.0 \%$ & $97.0 \%$ \\
\hline
\end{tabular}

a Excluding the ICA.

tion of pooled estimates of results from different research studies that represent the population studied. Overall, another significant advantage is to identify the gaps in the current knowledge in this research field to support further scientific efforts.

Detailed review of the research studies (Tables 4 and 5) included in this meta-analysis revealed study bias and limitations. Several research studies could not be included in the statistical analysis because insufficient data to create a $2 \times 2$ table were reported for CTA ${ }^{20-22,24}$ and CTP..$^{827,29-33}$ Other studies did not report the sensitivity, specificity, PPV, and NPV of CTA ${ }^{23}$ and CTP. ${ }^{8,29,32}$ Several biases were identified in many of the research studies, such as disease progression bias and review bias in interpreting both the index test (CTA or CTP) and the criterion standard (DSA). Given that many of the research studies had a time interval of $>12$ hours between the index test (CTA or CTP) and DSA, disease-progression bias could be present due to the temporal variation of vasospasm. Review bias may have also been present because the findings of either the index test or the criterion standard were determined with knowledge of the other test. A basic premise of comparing a new technique with the criterion standard is that the reviewers are blinded to the other test. These biases in the study design may significantly impact the internal validity of these research studies and, therefore, bring into question the quality of the results and conclusions.

In critically evaluating these research studies, a key element is to assess the characteristics of the study populations. For example, most of the research studies included in the metaanalysis performed CTA and CTP imaging on patients with symptoms of vasospasm. However, Ionita et $\mathrm{al}^{35}$ evaluated mostly asymptomatic patients in their study, reporting lower sensitivity and specificity of CTA for detecting vasospasm. This variability in the study populations of the different research studies has implications for their reported diagnostic accuracy of CTA and CTP. The characteristics of the study population should ideally represent the ASAH population for which CTA and CTP is intended for use in clinical practice.
Otherwise, the external validity of the study is jeopardized, and the results may not be translatable to other ASAH populations. Therefore, another methodologic advantage of a meta-analysis is pooling all the different study populations, including both symptomatic and asymptomatic patients, in the meta-analysis calculations, which improves the generalizability of its results.

There are also statistical advantages in performing a metaanalysis by using summary statistics that represent unbiased pooled estimates of diagnostic accuracy. The pooled estimates for CTA in the diagnosis of vasospasm were $79.6 \%$ sensitivity and $93.1 \%$ specificity, which included data from 6 research studies that had a total sample size of 141 patients and 1936 arterial segments. However, the results of this meta-analysis were most influenced by the largest study, performed by Chaudhary et al, ${ }^{19}$ which included 33 patients and reported $63 \%$ sensitivity. The authors accounted for this low sensitivity of vasospasm detection due to the early time point that CTA was performed, at 3 days after admission, which is before the peak incidence of vasospasm occurrence. As a result, there was a low incidence of moderate or severe vasospasm among their patients and a relatively low sensitivity and specificity of CTA compared with other studies. On the other hand, the pooled estimates for CTP in the diagnosis of vasospasm were $74.1 \%$ sensitivity and $93.0 \%$ specificity, which included only 3 research studies that had a total sample size of 64 patients. These results are not considered conclusive because they are based on limited data with high inconsistency index values.

If we go 1 step further, DORs were also calculated because they are not dependent on disease prevalence. High DORs for CTA of 124.5 and CTP of 43.0 were calculated. However, the SROC model is the most appropriate summary statistic to use in this meta-analysis because of the diagnostic threshold variation among the research studies. The overall SROC curve demonstrates the high diagnostic accuracy of CTA and CTP with AUCs of $98 \pm 2.0 \%$ and $97 \pm 3.0 \%$, respectively. The CTP analysis is based on only 3 studies; therefore, the SROC curve is of limited value and the $95 \%$ CI was not calculated, representing initial preliminary findings.

There are several limitations in our meta-analysis, including the limited number of relevant research studies for the statistical analysis because of incomplete data reported, high variability in the methodology, and heterogeneity of the data. The effects of these limitations are apparent in the wide 95\% CI for the SROC curve. Another limitation is that we confined our literature search to publications available in the English 
language only, though informal searching of the non-English publications did not demonstrate evidence that would have significantly changed our results. Additional limitations exist in comparing CTP with DSA because these 2 techniques measure different parameters. In the setting of vasospasm, DSA assesses the anatomic narrowing of cerebral arteries, whereas CTP assesses altered brain perfusion. Although there is a correlation between these 2 parameters, differences may be seen at the microcirculatory level reflecting perfusion changes on CTP with normal DSA examination findings. ${ }^{33,36}$

Diagnostic limitations have also been reported using CTP because of limited anatomic coverage with current techniques, beam-hardening artifact from clips and coils, and limited evaluation of the posterior fossa vascular territories. ${ }^{18,27}$ Patient risks of CTA and CTP are principally related to the administration of intravenous contrast (eg, contrast reaction, nephrotoxicity) and the effects of radiation exposure. However, when CTA and CTP are performed by using standard guidelines and techniques, then the possible benefits of early diagnosis and treatment of vasospasm outweigh these potential risks for patients with ASAH.

\section{Conclusions}

The appropriate use of CTA and/or CTP imaging for vasospasm diagnosis in patients with ASAH has the potential to impact patient care. In attempts to improve patient outcomes, emphasis has been placed on earlier and more accurate diagnosis of vasospasm to initiate prompt treatment to prevent the devastating sequelae of permanent neurologic deficits, infarction, and death. This meta-analysis provides a comprehensive critical review of the literature to date and statistical analysis of the current diagnostic accuracy of CTA and CTP for vasospasm in patients with ASAH. As stated above in the "Discussion," the following are the major points revealed from this meta-analysis: 1) The high diagnostic accuracy determined for both CTA $(98 \pm 2.0 \%)$ and CTP $(97 \pm 3.0 \%)$ is considered an initial preliminary finding derived from the current literature suggesting that CTA and CTP are potentially valuable techniques in patients with ASAH. 2) There were few research studies that met inclusion criteria for this meta-analysis, highlighting the need for future larger prospective studies to be performed. 3) The high variability in methodologic quality and reporting methods demonstrates the importance for future studies to adhere to the STARD criteria to reduce study bias and limitations. It is important to critically evaluate the current evidence to gain knowledge and understanding in appropriately using these new techniques in clinical practice today and to guide future scientific effort in this research field. Awareness of these results has the potential to impact patient care by providing supportive evidence for more effective use of CTA and CTP imaging in patients with ASAH.

\section{References}

1. Bederson JB, Connolly ES Jr, Batjer HH, et al. Guidelines for the management
of aneurysmal subarachnoid hemorrhage: a statement for healthcare profes-
sionals from a special writing group of the stroke council, American Heart
Association. Stroke 2009;40:994-1025. Epub 2009 Jan 22
2. van Gijn J, Kerr RS, Rinkel GJ. Subarachnoid haemorrhage. Lancet 2007;369:
306-18
3. Sanelli PC, Ougorets I, Johnson CE, et al. Using CT in the diagnosis and man- agement of patients with cerebral vasospasm. Semin Ultrasound CT MR 2006;27:194-206

4. Janjua N, Mayer SA. Cerebral vasospasm after subarachnoid hemorrhage. Curr Opin Crit Care 2003;9:113-19

5. Higgins J, Green S, eds. Cochrane Handbook for Systematic Reviews of Interventions. Cambridge, UK: The Cochrane Collaboration; 2008

6. Whiting P, Rutjes AW, Reitsma JB, et al. The development of QUADAS: a tool for the quality assessment of studies of diagnostic accuracy included in systematic reviews. BMC Med Res Methodol 2003;3:25

7. Laslo AM, Eastwood JD, Pakkiri P, et al. CT perfusion-derived mean transit time predicts early mortality and delayed vasospasm after experimental subarachnoid hemorrhage. AJNR Am J Neuroradiol 2008;29:79-85

8. Nabavi DG, LeBlanc LM, Baxter B, et al. Monitoring cerebral perfusion after subarachnoid hemorrhage using CT. Neuroradiology 2001;43:7-16

9. Glas AS, Lijmer JG, Prins MH, et al. The diagnostic odds ratio: a single indicator of test performance. J Clin Epidemiol 2003;56:1129-35

10. Deville WL, Buntinx F, Bouter LM, et al. Conducting systematic reviews of diagnostic studies: didactic guidelines. BMC Med Res Methodol 2002;2:9

11. DerSimonian R, Laird N. Meta-analysis in clinical trials. Control Clin Trials 1986;7:177-88

12. Higgins JP, Thompson SG, Deeks JJ, et al. Measuring inconsistency in metaanalyses. BMJ 2003;327:557-60

13. Deeks JJ. Systematic reviews in health care: systematic reviews of evaluations of diagnostic and screening tests. BMJ 2001;323:157-62

14. Walter SD. Properties of the summary receiver operating characteristic (SROC) curve for diagnostic test data. Stat Med 2002;21:1237-56

15. Moses LE, Shapiro D, Littenberg B. Combining independent studies of a diagnostic test into a summary ROC curve: data-analytic approaches and some additional considerations. Stat Med 1993;12:1293-316

16. Zamora J, Abraira V, Muriel A, et al. Meta-DiSc: a software for meta-analysis of test accuracy data. BMC Med Res Methodol 2006;6:31

17. Anderson GB, Ashforth R, Steinke DE, et al. CT angiography for the detection of cerebral vasospasm in patients with acute subarachnoid hemorrhage. AJNR Am J Neuroradiol 2000;21:1011-15

18. Binaghi S, Colleoni ML, Maeder $\mathrm{P}$, et al. CT angiography and perfusion CT in cerebral vasospasm after subarachnoid hemorrhage. AJNR Am J Neuroradiol 2007;28:750-58

19. Chaudhary SR, Ko N, Dillon WP, et al. Prospective evaluation of multidetector-row CT angiography for the diagnosis of vasospasm following subarachnoid hemorrhage: a comparison with digital subtraction angiography. Cerebrovasc Dis 2008;25:144-50

20. Joo SP, Kim TS, Kim YS, et al. Clinical utility of multislice computed tomographic angiography for detection of cerebral vasospasm in acute subarachnoid hemorrhage. Minim Invasive Neurosurg 2006;49:286-90

21. Ko NU, Vates GE, Smith WS. Correlation of CT angiography in vasospasm secondary to aneurysmal subarachnoid hemorrhage. Neurology 2003;60: A230-31

22. Ochi RP, Vieco PT, Gross CE. CT angiography of cerebral vasospasm with conventional angiographic comparison. AJNR Am J Neuroradiol 1997;18: 265-69

23. Otawara Y, Ogasawara K, Ogawa A, et al. Evaluation of vasospasm after subarachnoid hemorrhage by use of multislice computed tomographic angiography. Neurosurgery 2002;51:939-42, discussion 942-43

24. Takagi R, Hayashi H, Kobayashi H, et al. Three-dimensional CT angiography of intracranial vasospasm following subarachnoid haemorrhage. Neuroradiology 1998;40:631-35

25. Wintermark M, Ko NU, Smith WS, et al. Vasospasm after subarachnoid hemorrhage: utility of perfusion CT and CT angiography on diagnosis and management. AJNR Am J Neuroradiol 2006;27:26-34

26. Yoon DY, Choi CS, Kim KH, et al. Multidetector-row CT angiography of cerebral vasospasm after aneurysmal subarachnoid hemorrhage: comparison of volume-rendered images and digital subtraction angiography. AJNR Am J Neuroradiol 2006;27:370-77

27. Harrigan MR, Magnano CK, Guterman LR, et al. Computed tomographic perfusion in the management of aneurysmal subarachnoid hemorrhage: new application of an existent technique. Neurosurgery 2005;56:304-16

28. Moftakhar R, Rowley HA, Turk A, et al. Utility of computed tomography perfusion in detection of cerebral vasospasm in patients with subarachnoid hemorrhage. Neurosurg Focus 2006;21:E6

29. Pham M, Weigand A, Schlake HP, et al. Qualitative perfusion-computed tomography and vasospasm-related infarction after subarachnoid hemorrhage. Clin Neuroradiol 2006;16:41-46

30. Sviri GE, Britz GW, Lewis DH, et al. Dynamic perfusion computed tomography in the diagnosis of cerebral vasospasm. Neurosurgery 2006;59: 319-25

31. Sviri GE, Mesiwala AH, Lewis DH, et al. Dynamic perfusion computerized tomography in cerebral vasospasm following aneurysmal subarachnoid hemorrhage: a comparison with technetium-99m-labeled ethyl cysteinate 
dimer-single-photon emission computerized tomography. J Neurosurg 2006;104:404-10

32. Kanazawa R, Kato M, Ishikawa K, et al. Convenience of the computed tomography perfusion method for cerebral vasospasm detection after subarachnoid hemorrhage. Surg Neurol 2007;67:604-11

33. Wintermark M, Dillon WP, Smith WS, et al. Visual grading system for vasospasm based on perfusion CT imaging: comparisons with conventional angiography and quantitative perfusion CT. Cerebrovasc Dis 2008;26:163-70

34. Claassen J, Bernardini GL, Kreiter K, et al. Effect of cisternal and ventricular blood on risk of delayed cerebral ischemia after subarachnoid hemorrhage: the Fisher scale revisited. Stroke 2001;32:2012-20

35. Ionita CC, Graffagnino C, Alexander MJ, et al. The value of CT angiography and transcranial Doppler sonography in triaging suspected cerebral vasospasm in SAH prior to endovascular therapy. Neurocrit Care 2008;9:8-12

36. Murphy AM, Xenocostas A, Pakkiri P, et al. Hemodynamic effects of recombinant human erythropoietin on the central nervous system after subarachnoid hemorrhage: reduction of microcirculatory impairment and functional deficits in a rabbit model. J Neurosurg 2008;109:1155-64 